\title{
Cosmological decrease in brightness and angular broadening in the ionized inter-galactic medium detected in the MASIV quasar survey
}

Barney Rickett* ${ }^{\dagger}$ U California San Diego : E-mail: bjrickett@ucsd.edu

Jim Lovell , U Tasmania ; E-mail: Jim. Lovell@utas .edu . au

J-P Macquart, CalTech and NRAO ; E-mail: jpmeastro.caltech.edu

Dave Jauncey, ATNF Canberra ; E-mail: David.Jauncey@csiro.au

Hayley Bignall , JIVE, ASTRON ; E-mail: bignall@jive.nl

Lucyna Kedziora-Chudczer , ATNF Epping ; E-mail:

Lucyna.Kedziora-Chudczereatnf.csiro.au

Roopesh Ojha , USNO/NVI ; E-mail: rojha@usno.navy.mil

Tapio Pursimo, NOT ; E-mail: tpursimo@not.iac.es

Stas Shabala, MRAO, Cambridge ; E-mail: ss571@mrao.cam.ac.uk

Cliff Senkbeil , U Tasmania ; E-mail: cliffseutas.edu.au

\begin{abstract}
The MASIV survey of 482 compact sources at $6 \mathrm{~cm}$ found that half of them exhibit low level interstellar scintillation on timescales of two days. The ISS amplitude is quantified by the structure function of the flux density at a time lag of $2 \mathrm{~d}$, which is found to decrease with redshifts in the range 2-4. A structure function model is given depending on the fraction of flux density and angular diameter in the most compact core in each source. If the core emission is limited to a maximum brightness temperature, the minimum diameter should increase as $(1+z)^{0.5}$. However the observed decrease in ISS is significantly faster than this prediction. We consider a possible explanation in terms of angular broadening in the ionized IGM, based on optical observations of the $\operatorname{Ly} \alpha$ forest.
\end{abstract}

From Planets to Dark Energy: the Modern Radio Universe

October 1-5 2007

The University of Manchester, $U K$

\footnotetext{
*Speaker.

${ }^{\dagger}$ with support by US NSF grant AST 0507713
} 


\section{Interpretation of the MASIV Survey}

The Micro-Arcsecond Scintillation-Induced Variability (MASIV) survey of rapid variations in $6 \mathrm{~cm}$ flux density of flat spectrum compact radio sources was conducted in four 3 or 4 day observing epochs over a year. [7] reported results from the first epoch and jau2007 reported the results from all four epochs, in which over half of the 482 flat spectrum compact radio sources observed showed low level variability on time scales of 3 days or less. The cause of the variations was shown to be interstellar scintilaton (ISS), which requires the sources to have a compact "core" of angular size $\lesssim 50 \mu$ as. Most interestingly, it was found that the fraction of sources showing ISS decreases with redshift above about 2 . Here we attempt to interpret this important result.

We first describe the use of the structure function to quantify the amplitude and time scale of the variations. Since in the 4 epochs we only observed each source at intervals of $2 \mathrm{hr}$ over $3-4 \mathrm{~d}$, we cannot determine time scales shorter than $2 \mathrm{hr}$ or longer than $3 \mathrm{~d}$. Here we characterize the variance in each "light curve" by the structure function at a time lag of $t=2 \mathrm{~d}$ :

$$
D(t)=\frac{1}{N_{t}} \Sigma^{j, k}\left(S_{j}-S_{k}\right)^{2}
$$

where $S_{j}$ is a flux density measurement normalized by the mean flux density of the source over all 4 epochs and $N_{t}$ is the number of pairs of flux densities with a time lag $t$ binned in 2-hr increments. The structure function provides a statistically reliable estimator which can be modelled even for short data spans. We combined all 4 epochs to improve the statistics of each estimate and subtracted the estimated contribution due to noise and calibration errors. We then fitted a simple curve to each one and estimated $D(t=2 \mathrm{~d})$ and the time scale $\tau_{\text {iss }}$, which is defined by the time interval at which $D(t)$ would reach half of its saturation value that would be obtained at very large time lags. For the fastest variations $D(t)$ rises quickly to a saturated value and we classified the variations as fast if $\tau_{\text {iss }} \leq 0.5 \mathrm{~d}$. In the many cases of slow variations we see no saturation in $D(t)$ and can only set a lower limit of $3 \mathrm{~d}$ on $\tau_{\text {iss. }}$. The time scale is then classified as medium for estimates betwen 0.5 and $3 \mathrm{~d}$. No time scale was estimated for sources for which $D(t=2 \mathrm{~d})<0.0002$, i.e. $2 \mathrm{~d}$ difference in flux density less than $1 \% \mathrm{rms}$. The upper panel of Figure 1 shows a scatter plot of $D(t=2 \mathrm{~d})$ for the 335 sources for which we have redshifts [10]. We now consider why and how the structure function might depend on redshift.

In order to model $D(t=2 \mathrm{~d})$, we need to understand both the irregular electron density structure in the interstellar medium (ISM) and the angular sturcture of the most compact parts of flat spectrum sources. Whereas pulsars behave as point sources and exhibit 100\% ISS on times as short as minutes, even the most compact extra-galactic sources have diameters large enough to heavily quench their ISS, which reduces the ISS amplitude and lengthens its time scale. The low level variations on a few days of many flat spectrum sources was discovered by Heeschen [2], who called it flickering, and showed it to be due to ISS from its dependence on Galactic latitude [3].

Using the method developed for the ISS of quasar B0917+624 [12], which assumes a Kolmogorov ISM and Gaussian core for the quasar, we can approximate the structure function for a source large enough to substantially quench its ISS as:

$$
D(t) \sim 2 x^{2} m_{\theta}^{2} \frac{t^{a}}{t^{a}+\tau_{\theta}^{a}} ; m_{\theta} \sim\left[\frac{\theta_{\mathrm{F}}}{\sqrt{\theta_{\mathrm{F}}^{2}+\theta_{\mathrm{src}}^{2}}}\right]^{7 / 6} ; \tau_{\theta} \sim \frac{L}{V} \sqrt{\theta_{\mathrm{F}}^{2}+\theta_{\mathrm{src}}^{2}} .
$$




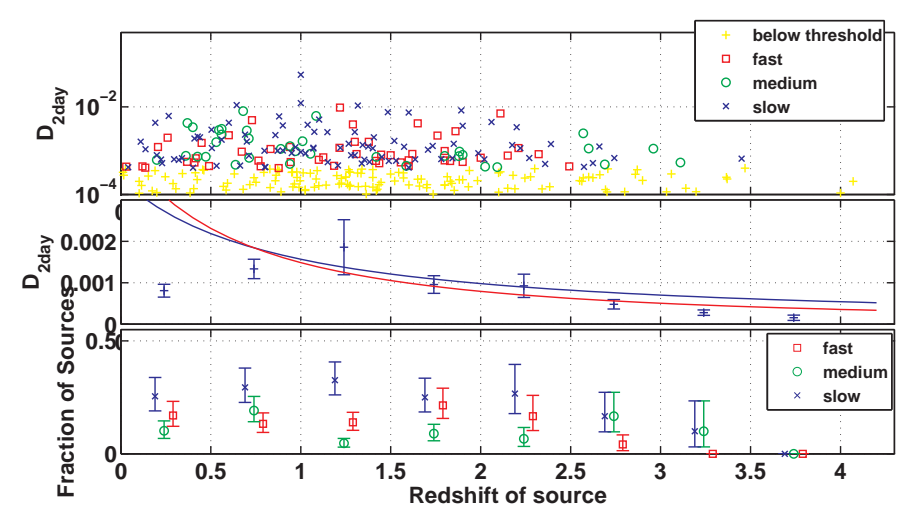

Figure 1: Upper panel: Scatter plot of $D(2 \mathrm{~d})$ against source redshift. The different symbols represent the three classifications of ISS timescale, as described in the text. Sources below the threshold of 0.0002 (weak variation) are plotted as plus signs Center panel: Mean value of $D(2 \mathrm{~d})$ in redshift bins (excluding extreme IHV quasar J1819+3845). Note lower levels of ISS at high redshift. The lines are theoretical relations described in the text. Lower panel: Fraction of sources in each timescale class in each bin. Note that fast variables decrease wih redshift more quickly than medium and slow variables.

Here $x$ is the fraction of the source flux density in a compact core of angular size $\theta_{\text {src }}, \theta_{\mathrm{F}}=$ $\sqrt{\lambda /(2 \pi L)}$ is the angular size subtended by the Fresnel scale in a scattering region at distance $L$, and $a$ is a constant $(1 \leq a \leq 2)$ that depends on the density distribution in the local ISM, which we here set to unity (see [13]). In this expression the scintillation index (rms/mean) for a point source is taken to be $100 \%$ assuming that $5 \mathrm{GHz}$ is near the transition from weak to strong ISS and $V$ the velocity of the Earth relative to the scattering region is assumed to be typically $30-50 \mathrm{~km} / \mathrm{s}$.

Under the conditions of the MASIV survey with a typical scattering distance of $L=500 \mathrm{pc}$, the ISS is heavily suppressed $\left(\theta_{\mathrm{src}}>\theta_{\mathrm{F}}\right)$ which reduces

$$
D(t=2 \mathrm{~d}) \sim x^{2}\left(\theta_{\mathrm{src}} / 7 \mu \operatorname{arcsec}\right)^{-7 / 3}\left(1+L \theta_{\text {src }} / V 2 \mathrm{~d}\right)^{-1} .
$$

This shows that $D(2 \mathrm{~d})$ decreases strongly with increasing source diameter, with the bracketed factor causing a further decrease for ISS time scales long compared to $2 \mathrm{~d}$. Applying this to Figure 1 we conclude that the AGNs and quasars that constitute the MASIV sample have angular diameters that increase substantially beyond a redshift of about 2. The bottom panel of that Figure shows that the fraction of fast scintillators decreases more rapidly with redshift than the fractions of medium and slow scintillators. This reinforces the idea that there are fewer very compact sources beyond $z=2$, since the fast scintillators must have smaller angular diameters than medium or slow scintillators.

The well-known " $\theta-z$ " relation would be one way of modelling this phenomenon, however, it is based on the model of an emitter of fixed length viewed versus redshift. A better physical model is that the sources are the compact cores of beamed jets pointing nearly toward the Earth. The MASIV sources were selected to have flat or inverted radio spectra which implies that the most compact emission is self-absorbed and so limited by a maximum brightness temperature (e.g. $[11,6])$. Consequently we consider a model in which the typical compact core has fraction $x$ of its flux density in a component with a maximum brightness temperature $T_{\mathrm{b}, \text { comov }}$ in the "comoving frame", which may be boosted by the Doppler factor of the jet above the limits imposed by 
synchrotron self-absorption or inverse Compton losses. $\theta_{\text {src }}$ is the apparent diameter as the waves enter the ISM which can be related to the observed compact flux density and brightness temperature $T_{\mathrm{b}, \mathrm{obs}}$. Since the latter will be reduced by cosmological expansion to $T_{\mathrm{b}, \mathrm{obs}}=T_{\mathrm{b}, \text { comov }} /(1+z)$, we model $\theta_{\text {src }}=\theta_{\text {emit }} \sqrt{1+z}$. We now assume no redshift evolution of the co-moving source quantities $\left(\theta_{\text {emit }}\right.$ and $\left.x\right)$ and obtain $D(2 \mathrm{~d}) \propto(1+z)^{-7 / 6}$. In the middle panel of Figure 1 we plot a curve of this form scaled to pass through the points near $z=2$, since the co-moving source quantities are not known.

While it is clear that the curve is a poor fit to the observations at both low and high redshifts, we are most concerned with understanding the high redshift deficit. It states that the typical angular diameters of these AGN sources increase between a redshift of 2.25 and 3.75 by more than is predicted assuming a fixed maximum brightness temperature and simple cosmological expansion. The pairs of horizontal lines in the left panel of Figure 2 show the observed $D(2 \mathrm{~d})$ as upper and lower error bounds at these redshifts. The solid and dashed curves are from equation (1.2) with the unknown compact fraction as a parameter (blue for $z=2.2$ and red for $z=3.7$ ). The intersection of the lines and curves determine co-moving source diameter $\theta_{\text {emit }}$. For example, with a $10 \%$ compact fraction the blue and red arrows give the allowed ranges in $\theta_{\mathrm{emit}}$.

We conclude that $\theta_{\text {emit }}$ increases by a factor of two from $z=2.2$ to $z=3.7$ and that $0.02<x<1$ is typical of these sources. With the smallest allowable $x \sim 0.02$ the observations require $\theta_{\text {emit }} \gtrsim$ $5 \mu$ as at $z=3.7$, corresponding to an observed minimum diameter $\theta_{\text {src }} \gtrsim 10 \mu$ as at that redshift. The increase in angular diameter with redshift could be due to evolution of the jet sources or the effects of propagation though the intergalactic medium (IGM). We now discuss the latter interpretation.

\section{Angular Broadening in the Ionized Inter-Galactic Medium}

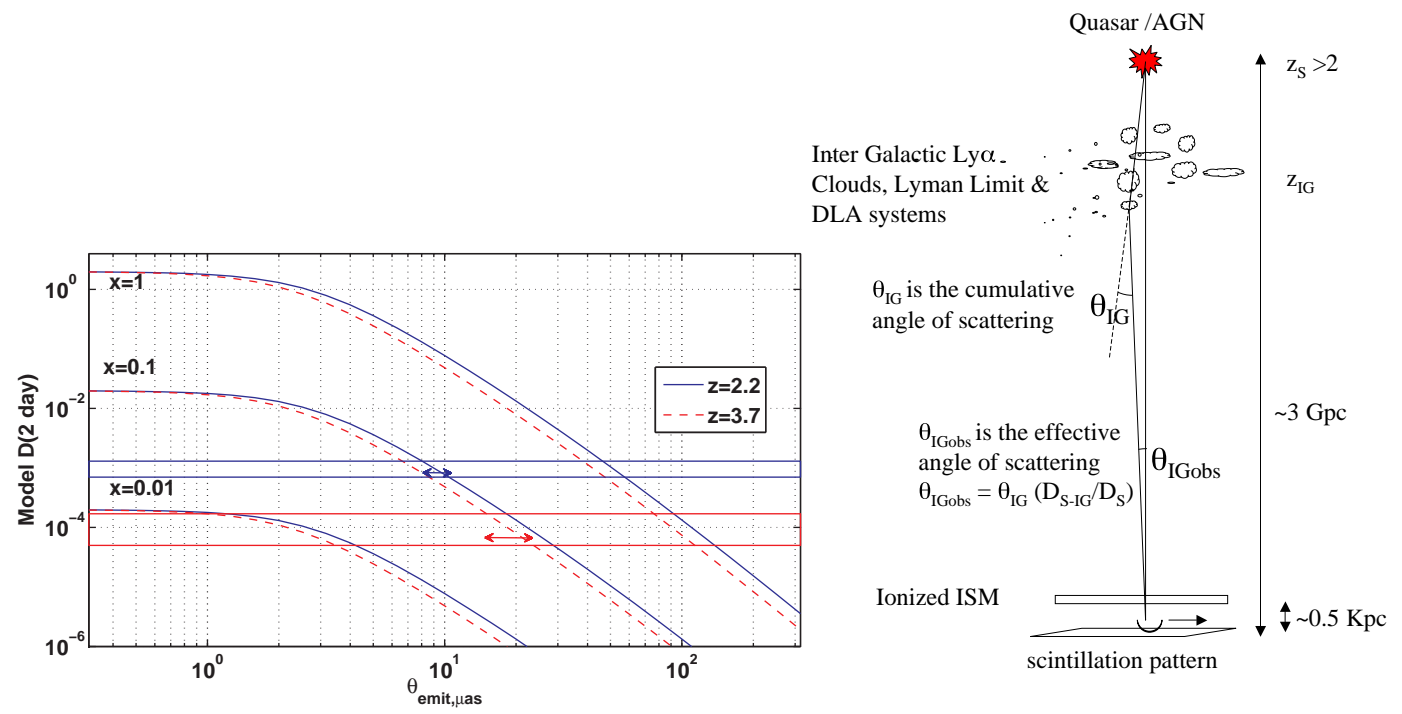

Figure 2: Left: Theoretical relation of $D(2 \mathrm{~d})$ to the emitted source diameter for redshifts redshifts of 2.25 and 3.75 with the compact fraction $x$ as a parameter. Right: Geometry of Inter-Galactic Scattering.

Angular broadening of radio waves as they propagate through the IGM could cause their apparent diameters to increase with redshift. Here we give a brief discussion of this effect due to 
random density fluctuations in the ionized IGM (IIGM), but recognize that random gravitational lensing might also be important.

While the Ly $\alpha$ forest provides a wealth of observations on the neutral $\mathrm{H}$ content of the IGM, there is little observational evidence on the IIGM, which is thought to contain most of the baryons in the Universe under current cosmological models. The key observable from the Ly $\alpha$ forest is the column density of neutral $\mathrm{H} N_{\mathrm{HI}} \sim n_{\mathrm{HI}} L$, where $L$ is a typical size of the HI clouds. Its distribution function over a wide range in column density has been studied versus redshift by a number of authors $[8,9,4]$. However, our concern is the electron density $n_{e}$, which is thought to result from photo-ionization of the IGM by UV photons from quasars. Haardt and Madau [1] describe a cosmological model for this ionizing radiation, which maximizes the ionization rate for redshifts 23. Near this maximum and assuming ionization equilibrium, one obtains $n_{e} \sim \sqrt{4 n_{\mathrm{HI}}} \mathrm{cm}^{-3}$. Then if we consider the IGM as clouds of size $L$ with mean densities $n_{\mathrm{e} L}$ and $n_{\mathrm{HI}}$ we can relate $n_{\mathrm{e} L}$ to the HI column density by

$$
n_{\mathrm{e} L} \sim \sqrt{4 N_{\mathrm{HI}} / L} \mathrm{~cm}^{-3} .
$$

In order to predict the angular broadening in these ionized clouds we have to make several further assumptions. We assume the plasma in the clouds is itself turbulent, similar to the condition in our own Galaxy, such that $n_{e}$ exhibits a fully developed Kolmogorov spectrum with $\delta n_{e} \sim n_{\mathrm{e} L}$ and outer scale $\sim L$. From this we can predict the rms angle of scattering by a single cloud at redshift $z$ for a source at redshift $z_{s}$ :

$$
\theta_{\mathrm{ig}} \sim 0.46 \lambda^{2.2} r_{\mathrm{e}}^{1.2}<n_{\mathrm{e} L}>^{1.2} L^{0.2}
$$

For wavelength $\lambda$ at the cloud this gives the (very small) deflection angle in radians at the cloud. In addition to the reduction from $\lambda=\lambda_{\mathrm{obs}} /(1+z)$, the angle we observe is reduced by the ratio of angular diameter distance between source and cloud and the distance between source and observer (see right panel of Figure 2).
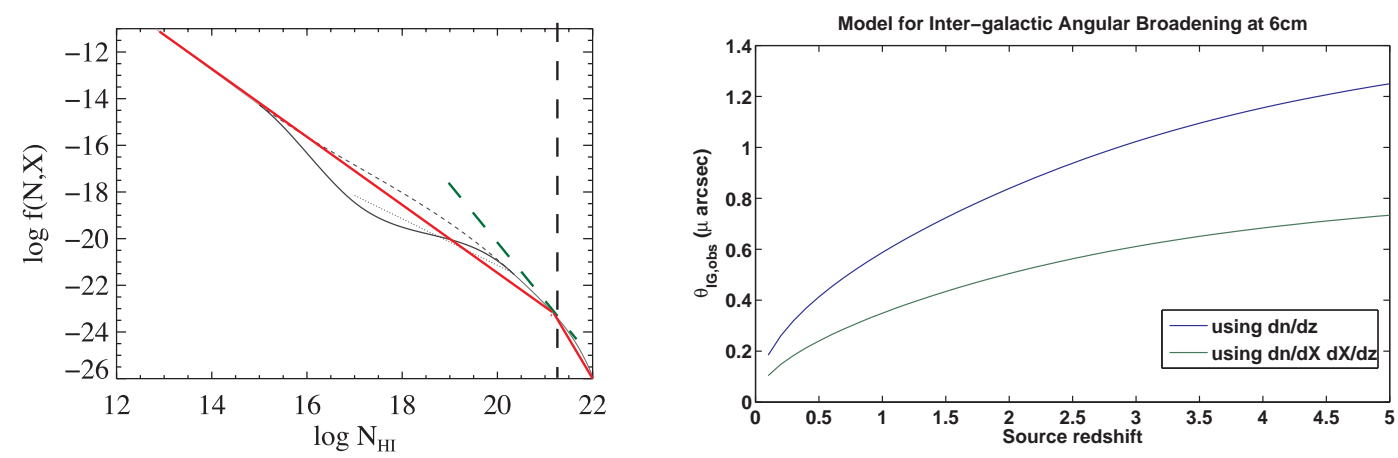

Figure 3: Left: Models for the distribution function of HI column densities from [9]; the dashed line with slope -2.2 is tangent to the model at the vertical dashed line $N_{\mathrm{HI}} \sim 2 \times 10^{21} \mathrm{~cm}^{-2}$; the red line has slope -1.4. Right: Model Calculation of the angular broadening in the ionized IGM as a function of source redshift. See text for the many assumptions.

A line of sight to a source at $z_{s}$ will pass through many clouds of differing column density and redshift. Since these scatterings are independent we sum their squares weighted by the intersection probablity $d P=g\left(N_{\mathrm{HI}}, z\right) d N_{\mathrm{HI}} d z$. Observers have modelled their results by assuming $g$ to be the 
separable product of functions of $N_{\mathrm{HI}}$ and $z \cdot g=N_{\mathrm{HI}}^{-\beta} A(z) \mathrm{cm}^{2}$. Peebles [8] cites values appropriate for redshifts near 3 and column densities near $10^{14} \mathrm{~cm}^{-2}$, as $\beta=1.46, A(z)=5.5 \times 10^{6}(1+z)^{\gamma}$ with $\gamma=2.75$. Though these power law forms apply over limited ranges in both variables, we have used them in a numerical integration of the complete expression for the squared angle of scattering versus $z_{s}$

We use equation (2.1) to eliminate $n_{\mathrm{e} L}$ but in place of the factor 4 we insert the analytical model for the redshift dependence of the ionization rate given by [1]. The integrand over $N_{\mathrm{HI}}$ is the observed $g\left(N_{\mathrm{HI}}, z\right)$ times a factor $N_{\mathrm{HI}}^{1.2} L^{-0.8}$. So now we must assume a model for the cloud sizes $L$, which we boldly take to be $10 \mathrm{kpc}$ independent of redshift, since it is thought that the clouds themselves do not expand with the Hubble flow. The left panel of figure 3 shows a composite model for the dependence of $g$ on $N_{\mathrm{HI}}$ near $z=2.7$ ([9]). In view of the factor $N_{\mathrm{HI}}^{1.2}$, the integral is dominated by the largest $N_{\mathrm{HI}}$, for which the dependence is not steeper than $N_{\mathrm{HI}} \propto N_{\mathrm{HI}}^{-2.2}$. In the right panel of Figure 3 we plot the predicted angle of scattering versus the redshift of the source. The prediction $\sim 1 \mu$ as is far below the $10 \mu$ as lower bound for the minimum observed source diameter derived above. Further if the scattering is caused by the structures such as DLAs with the highest neutral column density, the low sky covering fraction of these objects means that their angular broadening will also apply over a small fraction of the sky.

\section{Conclusions}

MASIV sources show a decrease in ISS amplitude with redshift. The ISS amplitude is quantified by the structure function of the flux density at a time lag of $2 \mathrm{~d}$. We give a simple model for how this depends on the fraction of flux density and angular diameter of the most compact core in each source. If the core emission is limited to a maximum brightness temperature, the minimum diameter should increase as $(1+z)^{0.5}$. However the observed decrease in ISS is significantly faster than this prediction, implying a doubling in source diameter observed at $6 \mathrm{~cm}$ between redshift 2.2 and 3.7 and minimum of $10 \mu$ as for sources at redshift 3.7. We consider a possible explanation for the increase in terms of angular broadening in the ionized IGM, but find that a simple model based on the Ly $\alpha$ forest results is ten times too small to explain the observed decrease. However, the potential of investigating the unseen baryons in the IIGM makes it important to re-examine the many assumptions in the model, including the possible evolution in beamed emission from the AGN jets.

\section{References}

[1] Haardt, F. \& Madau, P. 1996, ApJ, 461, 20

[2] Heeschen, D.S. 1984, AJ, 89, 1111

[3] Heeschen, D.S. \& Rickett, B.J. 1987, AJ, 93, 589

[4] Janknecht, E., Reimers, D., Lopez, S., \& Tytler, D., 2006, A\&A, 458, 427

[5] Jauncey, D. L., Lovell, et al., MRU, 2007

[6] Kellermann, K. I. and Pauliny-Toth, I. 1981, Ann. Rev. Astron. Astroph., 19, 373

[7] Lovell, J. E. J., Jauncey, D. L., Bignall, H. E., et al., 2003, AJ., 126, 1699

[8] Peebles, P.J.E., Principles of Physical Cosmology,Princeton U Press, 1993

[9] Prochaska, J. Herbert-Fort \& Wolfe. A., 2005, ApJ, 635, 123

[10] Pursimo, T., Ojha, R., et al., 2007, in "Extragalactic Jets: Theory and Observation from Radio to Gamma Ray", eds. Travis Rector and Dave de Young, (in press)

[11] Readhead, A. C. S., 1994, ApJ, 426, 51

[12] Rickett, B. J., Quirrenbach, A., Wegner, R., Krichbaum, T. P. \& Witzel A. 1995, ApJ, 293, 479

[13] Rickett, B.J., Lazio, T.J.W., \& Ghigo, F.D. 2006 ApJ Suppl., 165, 439 